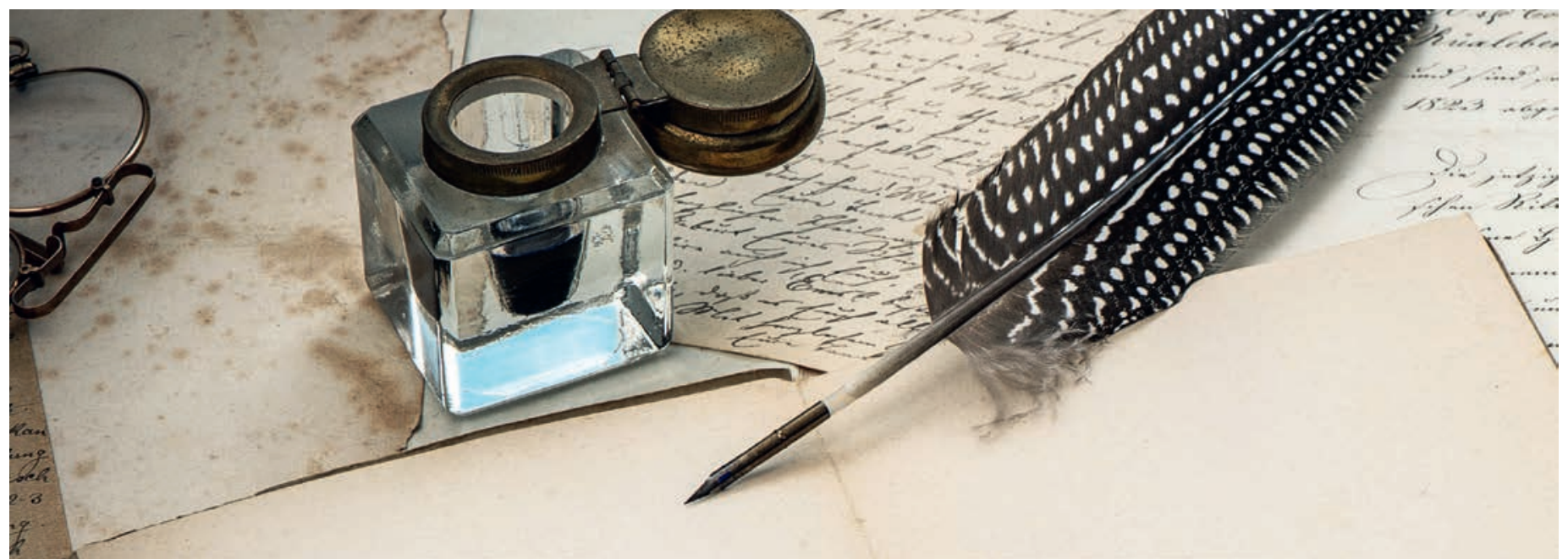

\title{
L'écriture, la potion magique du médecin
}

\section{Françoise Verrey Bass}

Dr méd., membre de la FMH

«...et tu crois vraiment que cela va marcher?» - «Je ne le crois pas seulement, j'en suis sûr!» Début de la conversation ce mardi matin entre mon calame (ma plume) et moi. Il faut dire que je suis arrivé à un point, où je serais presque d'accord d'essayer n'importe quoi, car j'en ai vraiment marre.

Je me présente: je suis médecin avec un cabinet en ville et à mes heures perdues j'écris des histoires. Non, ce n'est pas tout à fait exact. Je suis très organisé: je me lève tous les jours à 5 heures du matin pour avoir deux heures devant moi avant de commencer mes consultations, deux bonnes heures pour écrire dans la tranquillité de mon bureau, dans notre maison sur les hauteurs de la ville, avec vue plongeante jusqu'à la large rivière qui traverse la vallée actuellement verdoyante. Les montagnes d'en face ont leurs sommets encore enneigés de l'hiver qui s'est terminé il y a quelques semaines. C'est à ce moment de la journée, après une courte mais bonne nuit, que j'ai le plus d'idées et que ma plume parcourt gaiment la page blanche, la recouvrant de mon écriture assez régulière. Cette vieille collaboration avec la plume robuste héritée de mon grand-père m'a porté chance jusqu'à ces derniers temps. Les idées venaient à flots, en cascade, j'avais de la peine à suivre.
Mais depuis un mois, c'est le désert total dans ma tête, le vide complet. A cela s'ajoute l'angoisse de la page blanche. D'accord, quand je dis désert... c'est faux, il y a une vie intense quoique silencieuse dans les vrais déserts, ne serait-ce que les petits scarabées du matin et du soir qui surgissent tout à coup de partout et se mettent sur leurs petites pattes de derrière pour gober un peu de brume, le peu de liquide dont ils ont besoin pour vivre. Puis, au bout de quelques minutes, ils disparaissent, ils ne sont plus là, on ne les a pas vus s'enfoncer, tout va très vite.

Je réfléchis à cette scène, où, assis en haut d'une dune un matin de printemps après l'apparition des scarabées, je demandai à mon guide pourquoi ils se mettent sur leurs pattes de derrière. Celui-ci, l'œil rieur, me répondit que c'était leur manière de prier Allah et de le remercier d'avoir survécu à la nuit - puis au jour. Je pense à cela en roulant mon calame entre mes doigts. C'est le nom que j'ai donné à ma plume, un nom qui était usité au Moyen Age et qui me plaît beaucoup, qui va bien avec l'activité ample et élégante, souvent expectative de ma plume, contrairement au mot plume qui laisse plutôt penser à une plume d'oiseau qui court sur le papier. 
Donc, depuis un mois, je n'arrive plus à aligner un mot après l'autre sur la page blanche. Un vide total du cerveau.

Raison pour laquelle mon calame me propose une potion magique, de quoi faire redémarrer mon cerveau. "Je t'explique: tu mets un peu d'eau tiède dans une casserole, tu ajoutes une pincée de sel, tu fais chauffer le liquide, mais à peine, à peine. Puis, après avoir vidé mon réservoir, tu me couches dans ce bain, le temps que je dépose un exemplaire de mon alphabet dans l'eau..., non, deux; deux exemplaires, ce serait mieux, la potion te montera ainsi plus complètement et plus vite au cerveau.

Au bout de quelques minutes, 7 exactement, tu me sortiras de l'eau, me sécheras bien complètement, je ne veux pas rouiller, puis tu rempliras de nouveau mon réservoir. Ce dernier rempli, tu gicleras deux fois environ $0,5 \mathrm{ml}$ de ton encre bio dans l'eau. Pour améliorer la potion, tu ajouteras quantum satis de gingembre et de citron."

«Tu es folle! Tu vas m'empoisonner!» Ce n'est pas l'encre que je crains. Elle est vraiment biologique et de la région, mais heureusement pas biodégradable - sinon adieu veau, heu... Non, seulement tout ce que j'écris depuis deux décennies et demie! Elle est faite avec une décoction de plantes toutes comestibles que je vais chercher moi-même dans les prairies de la montagne au-dessus de notre maison. Elle a une belle couleur bleu-vert avec des tons irisés selon la façon dont elle est exposée à la lumière. Non, ce sont les lettres qui me font peur. Elles sont peut-être toxiques! Même si je suis sûr que ma plume ne vient ni des Etats-Unis, ni de Russie, ni de Syrie. C'est une bonne vieille plume made in Switzerland il y a environ 100 ans.

Alors là, ma plume se met à rire franchement: «Ce que tu peux être compliqué! Souviens-toi la manière dont tu as appris à lire à 5 ans. Votre maman vous faisait de la soupe aux lettres, de toutes petites pâtes en forme de lettres dans un bouillon. Tu les sortais avec ta cuillère et les alignais le long de l'assiette, les mettant soigneusement dans le bon sens. C'est ainsi que tu as appris l'alphabet. Ensuite, répétant le nom de chaque lettre, tu les mettais en bouche et les avalais...» «Stop!» Je l'interromps: «c'étaient de petites pâtes, pas des lettres sorties d'une plume à réservoir, tandis que tes lettres...» "Sont virtuelles, donc tu n'as absolument rien à craindre! En buvant cette potion magique qui va te monter vite fait au cerveau, tu n'auras plus qu'à secouer un peu la tête dans tous les sens et tes réservoirs d'idées seront de nouveau remplis, c'est aussi simple que cela!», conclut Calame sur un ton docte.

L'idée me travaille, fait son chemin. «Est-ce que l'on peut répéter l'opération si jamais? Chaque fois que l'on a une attaque de page blanche, un désert au cerveau?..." «Tu veux ma mort, ou bien? Non, rien à faire, car j'y laisse quand même pas mal de plumes, avec deux alphabets. Du reste, tu as pu écrire sans peine pendant 26 ans et demi, avec cette transfusion de matière première, tu vas facilement pouvoir en écrire encore autant, c'est-à-dire jusqu'à ta mort physique ou à celle prématurée de ton cerveau, due à une raison $\mathrm{x}$, une maladie quelconque...» «J'adore ton humour noir, merci, tu sais bien nous remonter le moral! D'une compagne si fidèle pendant tant d'années, j'aurais vraiment attendu plus d'affection, voire même de l'empathie pour mon désert blanc." "Mais que veux-tu de plus? L'empathie, c'est bien beau, mais là, cela ne t'aide pas. Tandis que moi, je te propose une vraie solution qui tient la route, qui va te remplir le cerveau, car mes deux alphabets vont se multiplier dès qu'ils auront atteint leur but, ton cerveau! Ils vont tourbillonner, tu n'auras plus qu'à attraper les mots au passage et à les assembler selon ton imagination, ton inspiration! Et de plus, c'est le plus beau cadeau que je puisse te faire, à comparer à une transplantation de tissu vivant. Je te donne une - d'accord, vu ainsi - assez modeste partie de moi-même, mais c'est ce que j'ai de plus précieux. Et tu n'auras même pas besoin de prendre des médicaments pour éviter un rejet, car les lettres virtuelles sont compatibles avec n'importe quel sujet. De plus, je suis ta fidèle compagne, nous sommes faits l'un pour l'autre! Donc, à toi d'y mettre ton âme, ton esprit, pour en faire une véritable potion magique!»

Là, Calame a vraiment frappé un grand coup. Je reste silencieux - et je pense que je vais peut-être quand même me décider à préparer, puis ensuite avaler cette potion magique. Qu'est-ce que je risque? Au pire, que cela ne marche pas du tout, que mon cerveau reste vide et blanc. Au mieux, Calame aura eu raison et elle se remettra à parcourir joyeuse la page blanche poussée par mes doigts impatients d'écrire tout ce qui se pressera à nouveau dans mon cerveau. Un bonheur matinal retrouvé, et si possible pour de longues années encore! Allez, on y va...

\section{Crédit photo}

() Liligraphie | Dreamstime.com (image prétexte) 